\title{
DISCRETE MODELS FOR SCATTERING POPULATIONS
}

\author{
PATRICK FAYARD $* * *$ AND \\ TIMOTHY R. FIELD, ${ }^{*}$ McMaster University
}

\begin{abstract}
Jakeman's random walk model with step number fluctuations describes the coherent amplitude scattered from a rough medium in terms of the summation of individual scatterers' contributions. If the scattering population conforms to a birth-death immigration model, the resulting amplitude is $K$-distributed. In this context, we derive a class of diffusion processes as an extension of the ordinary birth-death immigration model. We show how this class encompasses four different cross-section models commonly studied in the literature. We conclude by discussing the advantages of this unified description.
\end{abstract}

Keywords: Stochastic differential equation; scattering of waves; $K$-distribution; FokkerPlanck equation; population dynamics; diffusion process

2010 Mathematics Subject Classification: Primary 74J20

Secondary $93 \mathrm{E} 03$

\section{Introduction}

Advances in the modeling of electromagnetic scattering returns, especially from marine surfaces, were enabled by a fundamental mathematical tool, the random walk model [11], [12]. It takes the total scattered field as the coherent summation of (independent) individual scatterers' contributions. For a population driven by a birth-death immigration (BDI) process, the resulting scattered amplitude is $K$-distributed, and its dynamics can be derived in a stochastic dynamical framework [9]. More precisely, we can derive a stochastic differential equation (SDE) for the scattering radar cross section (RCS), defined as the continuous limit of such a population for an asymptotically large number of individual scatterers, whose asymptotic $\Gamma$-distribution, the socalled texture, gives rise to the $K$-distribution for the scattered intensity [8]. Radar engineers have postulated various other distribution models for this texture [1], [4]. Motivated by the mathematical basis of the $\Gamma$-texture provided by the BDI process, the present paper succeeds in deriving the other texture models considered in the literature on the basis of an underlying discrete population. This paves the way towards the discussion of the Pearson diffusions, a class of stationary Markov processes extensively studied in [18], which encompasses all the probability densities proposed for the RCS. We also discuss how the particular structure of the SDE associated with this class supports the viability of such probability distributions to represent the RCS. In particular, it is possible to derive a generic form for the intensity autocorrelation function, provided that the scattering cross-section process is a Pearson diffusion. Also of

Received 23 February 2010; revision received 22 November 2010.

* Postal address: Department of Electrical and Computer Engineering, McMaster University, 1280 Main Street West, Hamilton, Ontario L8S 4K1, Canada.

** Email address: fayardp@mcmaster.ca 
interest is the possibility to deduce the parameters of the underlying discrete population model from the intensity moments, which are observable.

\section{Population model}

\subsection{Random walk}

The electric field scattered from a rough surface may conveniently be represented by a random walk model [12]

$$
\varepsilon_{t}^{(N)}=\sum_{j=1}^{N} \exp \left[\mathrm{i} \varphi_{t}^{(j)}\right],
$$

that is, as the summation of individual scatterers' contributions with normalized amplitudes. A phase diffusion model takes $\left\{\varphi_{t}^{(j)}\right\}$ as a collection of (displaced) Wiener processes $\left\{W_{t}^{(j)}\right\}$ on a suitable time scale

$$
\varphi_{t}^{(j)}=\Delta^{(j)}+\mathscr{B}^{1 / 2} W_{t}^{(j)}
$$

with random initializations $\left\{\Delta^{(j)}\right\}$, a set of independent random variables uniformly distributed on the interval $[0,2 \pi)$. Consequently, an application of Itô's formula (cf. [14, Chapter 4]) to the normalized amplitude $\gamma_{t}=\lim _{N \rightarrow \infty} \varepsilon_{t}^{(N)} / N^{1 / 2}$ yields the following SDE [9]:

$$
\mathrm{d} \gamma_{t}=-\frac{1}{2} \mathscr{B} \gamma_{t} \mathrm{~d} t+\mathscr{B}^{1 / 2} \mathrm{~d} \xi_{t}
$$

At this stage, the number of scatterers is constant and the 'speckle' $\gamma_{t}$ is thus a complex OrnsteinUhlenbeck process with a modulus amplitude that is Rayleigh distributed. More sophisticated models may be obtained by allowing fluctuations in the population of scatterers, as considered next.

\subsection{First-order transitions}

Accordingly, let us consider a first-order master equation (cf. [15]) for the time evolution of the probability $P_{N}(t)=\mathrm{P}\left[N_{t}=N\right]$ for a continuous-time integer-valued population process (cf. Figure 1). Denoting the (time-dependent) generation and recombination rates by $G_{N}(t)$

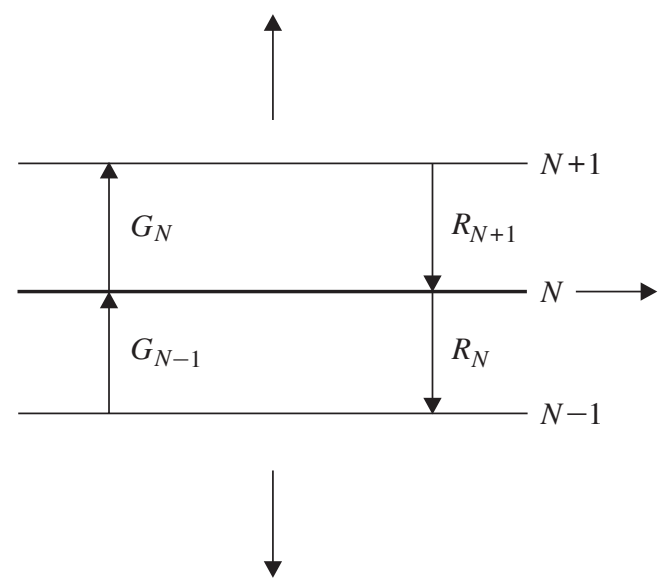

FIGURE 1: First-order transition process. 
and $R_{N}(t)$, respectively, we find from conservation of probability that

$$
\frac{\mathrm{d} P_{N}(t)}{\mathrm{d} t}=R_{N+1} P_{N+1}(t)+G_{N-1} P_{N-1}(t)-\left(G_{N}+R_{N}\right) P_{N}(t) .
$$

A widely used model for sea clutter, the $K$-distribution model [11], posits a BDI process for the scatterers' fluctuations, that is, linear generation and recombination rates $G_{N}=\lambda N+v$ and $R_{N}=\mu N$ with constant birth, death, and immigration rates $\lambda, \mu$, and $\nu$, respectively, yielding a time asymptotic population mean [2, Chapter 3]

$$
\bar{N}=\frac{v}{\mu-\lambda} .
$$

For an asymptotically large population $N_{t}$, it is instructive to consider the continuum limit $x_{t}=N_{t} / \bar{N}$ so that $x_{t}$ will account for the RCS. In effect, for a fluctuating number of steps, the (normalized) scattered amplitude $\psi_{t}=\lim _{N_{t} \rightarrow \infty} \varepsilon^{\left(N_{t}\right)} / \bar{N}^{1 / 2}$ has the following compound representation:

$$
\psi_{t}=\lim _{N_{t} \rightarrow \infty}\left[\left(\frac{N_{t}}{\bar{N}}\right)^{1 / 2} \frac{\varepsilon_{t}^{(N)}}{N_{t}^{1 / 2}}\right]=x_{t}^{1 / 2} \gamma_{t}
$$

This corresponds to the experimentally evidenced multiplicative representation of the scattered amplitude in terms of two (independent) components: the Gaussian speckle $\gamma_{t}$, modulated by (the square root of) the cross section $x_{t}$ [16]. In the continuum limit, the master equation (2.4) reduces to the Fokker-Planck equation (FPE) [8],

$$
\frac{\partial \mathcal{P}_{t}(x)}{\partial t}=\lambda \frac{\partial^{2}}{\partial x^{2}}\left[x \mathcal{P}_{t}(x)\right]+v \frac{\partial}{\partial x}\left[(x-1) \mathcal{P}_{t}(x)\right],
$$

through a Taylor expansion for $\mathcal{P}_{t}(x)=P_{\bar{N} x}(t)$. There exists an SDE associated with (2.7) (cf. Chapter 4 of [15]) in terms of the rescaled process $x \mapsto \alpha x$ for $\alpha=\nu / \lambda$ :

$$
\mathrm{d} x_{t}=-\mathcal{A}\left(x_{t}-\alpha\right) \mathrm{d} t+\left(2 \mathcal{A} x_{t}\right)^{1 / 2} \mathrm{~d} W_{t}^{(x)} .
$$

Here $W_{t}^{(x)}$ denotes a Wiener process. In the asymptotic limit, whose dynamics is represented by (2.8), the gamma texture of the $K$-distribution model is thus recovered.

\subsection{Pearson diffusions}

Let us now consider a class of (continuous-valued) diffusion processes: the Pearson diffusions [10]. Pearson diffusions are stationary processes with an SDE bearing a close resemblance to $(2.8)$ :

$$
\mathrm{d} x_{t}=-\mathcal{A}\left(x_{t}-m\right) \mathrm{d} t+\sqrt{2 \mathcal{A}\left(a x_{t}^{2}+b x_{t}+c\right)} \mathrm{d} W_{t}^{(x)} .
$$

Here $\mathcal{A}>0$ is a time scaling parameter, and $a, b$, and $c$ are the process' state parameters. The FPE corresponding to dynamics (2.9) has stationary solutions belonging to the Pearson class, as elucidated upon below. If it exists, the mean of the asymptotic distribution for $x_{t}$ is given by $\left\langle x_{t}\right\rangle=m$. The domain of $x_{t}$ is constrained to ensure that the square root in (2.9) is well defined. This condition may be fulfilled by setting $c=0$ (as in [4]), but we will consider a broader range of processes here. This process is a diffusion (rather than a mere Itô process) since the drift $b_{t}=m-x_{t}$ and volatility $\Sigma_{t}=a x_{t}^{2}+b x_{t}+c$ coefficients are state dependent 
(i.e. functions of $x_{t}$ ). Pearson diffusions may alternatively be defined through the following differential equation satisfied by their asymptotic distributions $W(x)$ :

$$
\frac{\mathrm{d} W(x)}{\mathrm{d} x}=-\frac{(2 a+1) x-m+b}{a x^{2}+b x+c} W(x) .
$$

Equation (2.10) forms the so-called Pearson system. The first investigation of the equivalence between the probability densities satisfying the Pearson system and the stationary distributions for processes represented by (2.9) was performed by Wong [18] via the FPE associated with (2.9).

From the compound representation (2.6), the dynamics of the scattered amplitude $\psi_{t}$ are uniquely determined by the dynamics of $x_{t}$. Keeping this in mind, the Pearson class is of interest since it incorporates a variety of probability distributions used by radar experimentalists to model the texture of a scattered amplitude conforming to this multiplicative representation. For a volatility coefficient given by $\Sigma=b x$, the RCS is asymptotically $\Gamma$-distributed with scale parameter $k$ and shape parameter $m / k$. The resulting scattered envelope will have a $K$-distribution where the usual parameters $b$ and $v$ are given by $b=1 / k$ and $v=m / k$. This distribution has been used for several decades to model sea clutter. For maritime radars, the normalized variance $R=\operatorname{var}[x] /\langle x\rangle^{2}=1 / v$ is related to the sea behavior. A large and small $R$ respectively represent a high and calm sea state. The shape parameter $v$ therefore depicts the sea state [17]. Another Pearson diffusion with volatility $\Sigma=a x^{2}$ yields a texture that has an inverse $\Gamma$-distribution with scale parameter $a / m$ and shape parameter $1+1 / a$. The resulting distribution for the envelope was shown to match well data from a lake clutter [1]. In a slightly different context (synthetic aperture radar interferometry), two additional Pearson diffusions were successfully confronted with real data [4] for $\Sigma=a x^{2}+b x$. In this case, the RCS distribution is a $\beta$-distribution of the first kind with parameters $m / b$ and $-(m / b+1 / a)$ if $b / a \leq 0$ or a $\beta$-distribution of the second kind with parameters $m / b$ and $1+1 / a$ if $b / a \geq 0$. Apart from these four instances of (2.9), other Pearson diffusions may prove adequate for scattering applications (whether the resulting distribution for the intensity is tractable or not).

\subsection{Associated population model}

We have described above the pertinence of the Pearson class for scattering applications. Motivated by the well-known derivation of (2.8) on the basis of a discrete population model in the continuum limit, we proceed to derive the Pearson processes on a similar basis of equivalent underlying discrete-valued population models.

To this end, let us first extend the BDI process as

$$
G=v+\lambda N+\bar{\epsilon} N^{2}, \quad R=\mu N+\bar{\epsilon} N^{2},
$$

where the nonnegative parameter $\bar{\epsilon}=\epsilon / \bar{N}$ is of order $\bar{N}^{-1}$ to ensure that the continuous limit of the resulting population is of diffusive type (cf. the discussion in [8, Section II]). Since the quadratic coefficients in the generation and recombination transition functions are equal, the BDI time asymptotic mean (2.5) is recovered (cf. Equation (10) of [3]). Following the same token as for a BDI process, if we substitute the transition rates (2.11) into (2.4) and consider a Taylor expansion, the SDE associated with the resulting FPE reads

$$
\mathrm{d} x_{t}=v\left(1-x_{t}\right) \mathrm{d} t+\sqrt{2\left(\lambda x_{t}+\epsilon x_{t}^{2}\right)} \mathrm{d} W_{t}^{(x)} .
$$

By virtue of Itô's calculus, the transformed cross section $x \mapsto m(x+p) /(1+p)$ is also a Pearson diffusion with ad hoc parameters, as captured by the following result, for constant $p$ identified below. 
Proposition 2.1. The class of Pearson diffusions, as embodied by (2.9), emerges as the affine transformed version $x \mapsto m(x+p) /(1+p)$ of the continuous limit of a discrete-valued population model with transition rates $G=\nu+\lambda N+\bar{\epsilon} N^{2}$ and $R=\mu N+\bar{\epsilon} N^{2}$, with

$$
\begin{aligned}
v & =\mathcal{A} \\
\epsilon & =\mathcal{A} a \\
\lambda & =\mathcal{A} \frac{b(1+p)}{m+2 a p}, \\
p & =\frac{-(2 c+m b)+m \sqrt{b^{2}-4 a c}}{2\left(a m^{2}+b m+c\right)},
\end{aligned}
$$

where $\mu$ is a free parameter that determines the asymptotic population mean $\bar{N}$ through (2.5).

\section{Application to scattering theory}

An appealing feature of the representation of the cross section via the SDE (2.9) is that the stochastic framework introduced in [9] and pursued in [7], originally for a $K$-distributed amplitude, may readily be extended to encompass the Pearson cross sections discussed above. These considerations could be applied to the cross-section inference or to the derivation of the spectral properties of the scattering amplitude.

\subsection{Intensity autocorrelation}

Owing to the linear drift in (2.9), the Pearson diffusions share the feature that their normalized covariance function is an exponentially decaying function of time, i.e.

$$
\left\langle\left(\frac{x_{t}-m}{\sigma}\right)\left(\frac{x_{0}-m}{\sigma}\right)\right\rangle=\exp [-\mathcal{A} t] .
$$

(The mean $m$ and the variance $\sigma^{2}$ in the above expression can be deduced from (3.3) below. Also, $x_{0}$ denotes the initial value drawn from the asymptotic distribution, thus ensuring stationarity of the process.) Meanwhile, from the compound expression (2.6), the scattered intensity $z_{t}=\left|\psi_{t}\right|^{2}$ has a multiplicative representation in terms of two independent processes, and its autocorrelation function is given by

$$
\left\langle z_{t} z_{0}\right\rangle=\left\langle u_{t} u_{0}\right\rangle\left\langle x_{t} x_{0}\right\rangle
$$

The dynamics of the process $u_{t}=\left|\gamma_{t}\right|^{2}$ proceed from (2.3) and read as

$$
\mathrm{d} u_{t}=\mathscr{B}\left(1-u_{t}\right) \mathrm{d} t+\sqrt{2 \mathscr{B} u_{t}} \mathrm{~d} W_{t}^{(u)},
$$

in which $\gamma_{t} \mathrm{~d} \xi_{t}^{*}+\gamma_{t}^{*} \mathrm{~d} \xi_{t}=\sqrt{2 \mathscr{B} u_{t}} \mathrm{~d} W_{t}^{(u)}$. As a consequence, $u_{t}$ also belongs to the class of Pearson diffusions and satisfies (3.1). As a result, for a speckle distribution satisfying the Pearson equation (2.10), the autocorrelation of the intensity reads

$$
\left\langle z_{t} z_{0}\right\rangle=\left(m^{2}+\sigma^{2} \exp [-\mathcal{A} t]\right)(1+\exp [-\mathscr{B} t]) .
$$

The normalized covariance of the intensity $z_{t}$ is the product of two exponentially decaying terms with timescales $\mathcal{A}^{-1}$ and $\mathscr{B}^{-1}$, respectively characteristic of $x_{t}$ and $u_{t}$ (in radar applications, 
$\mathcal{A}^{-1}$ is typically of the order of many seconds whereas $\mathcal{B}^{-1}$ is of the order of ten milliseconds [17, pp. 106-107]). We also readily observe that the intensity power spectral density is given by

$$
\begin{aligned}
S_{z}(\omega)= & m^{2} \delta(\omega)+\sigma^{2} \frac{\mathscr{A}}{2 \pi\left(\mathcal{A}^{2}+\omega^{2}\right)}+m^{2} \frac{\mathscr{B}}{2 \pi\left(\mathscr{B}^{2}+\omega^{2}\right)} \\
& +\sigma^{2} \frac{\mathscr{A}+\mathscr{B}}{2 \pi\left((\mathcal{A}+\mathscr{B})^{2}+\omega^{2}\right)} .
\end{aligned}
$$

Equation (3.2) generalizes the expression obtained in [9] for a $K$-distributed amplitude. We have thus encompassed the wider class of intensity distributions considered in [1] and [4] for which we have derived their autocorrelation and spectral properties.

\subsection{Deduction of the associated population model}

An interesting property concerning the moments is obtained by considering Itô's formula for the process $y_{t}=x_{t}^{n}$ :

$$
\begin{aligned}
\mathrm{d}\left(x_{t}^{n}\right)= & -\mathcal{A} n x_{t}^{n-1}\left(x_{t}-m\right) \mathrm{d} t+\mathcal{A} n(n-1) x_{t}^{n-2}\left(a x_{t}^{2}+b x_{t}+c\right) \mathrm{d} t \\
& +n x_{t}^{n-1} \sqrt{2 \mathcal{A}\left(a x_{t}^{2}+b x_{t}+c\right)} \mathrm{d} W_{t}^{(x)} .
\end{aligned}
$$

Taking the expectation removes the Brownian term and, for the moments of a Pearson diffusion, we obtain the recurrence relation

$$
\left\langle x_{t}^{n}\right\rangle=\frac{(m+(n-1) b)\left\langle x_{t}^{n-1}\right\rangle+(n-1) c\left\langle x_{t}^{n-2}\right\rangle}{1-(n-1) a},
$$

initialized with $\left\langle x_{t}^{0}\right\rangle=1$ and $\left\langle x_{t}^{1}\right\rangle=m$ (the $n$th moment exists for $a<1 /(n-1)$, as observed by investigating its existence for all the different types of Pearson diffusion [10]). On the other hand, for a compound Gaussian clutter, the moments of the RCS $x_{t}$, and of the intensity $z_{t}$, are related according to (see Chapter 4 of [17])

$$
\left\langle z_{t}^{n}\right\rangle=n !\left\langle x_{t}^{n}\right\rangle
$$

Conversely, if a probability distribution for the scattered intensity does not exhibit such a recurrence relation for the intensity moments (e.g. the Weibull distribution $\left\langle z^{n}\right\rangle=a^{n} \Gamma(n / \beta+$ $1)$ ), we cannot postulate a cross-section distribution belonging to the Pearson system (although a compound distribution resulting from (2.6) may exist). Combining (3.3) and (3.4) yields an expression for the Pearson diffusion parameters in terms of the first four moments of the intensity, if they exist, as exemplified by

$$
a=\left|\begin{array}{ccc}
\left\langle z_{t}^{2}\right\rangle-2\left\langle z_{t}\right\rangle^{2} & 2\left\langle z_{t}\right\rangle & 2 \\
\left\langle z_{t}^{3}\right\rangle-3\left\langle z_{t}^{2}\right\rangle\left\langle z_{t}\right\rangle & 6\left\langle z_{t}^{2}\right\rangle & 12\left\langle z_{t}\right\rangle \\
\left\langle z_{t}^{4}\right\rangle-4\left\langle z_{t}^{3}\right\rangle\left\langle z_{t}\right\rangle & 12\left\langle z_{t}^{3}\right\rangle & 36\left\langle z_{t}^{2}\right\rangle
\end{array}\right| /\left|\begin{array}{ccc}
\left\langle z_{t}^{2}\right\rangle & 2\left\langle z_{t}\right\rangle & 2 \\
2\left\langle z_{t}^{3}\right\rangle & 6\left\langle z_{t}^{2}\right\rangle & 12\left\langle z_{t}\right\rangle \\
3\left\langle z_{t}^{4}\right\rangle & 12\left\langle z_{t}^{3}\right\rangle & 36\left\langle z_{t}^{2}\right\rangle
\end{array}\right|
$$

In other words, the state parameters $a, b$, and $c$ of (2.9) and (2.10) can be extracted from the intensity moments. Since these state parameters also specify the discrete-valued underlying population model (via Proposition 2.1), it is possible to express the transition rates of the latter in terms of the intensity raw moments (which are directly observable). A precise knowledge of the underlying population model parameters provides some insight into the underlying physical phenomena. For instance, experimental data show that the scattering pattern depends on the 
incident wave polarization (see, e.g. [5]). Comparing the population parameters for different polarizations may improve the understanding of this discrepancy.

Besides the state parameters, knowledge of the characteristic frequency constant $\mathcal{A}$ is also required to fully determine the population model. This quantity may also be extracted from the observed time series of the intensity (as discussed in [6]). As a result, the discrete population model driving the speckle can be entirely inferred from the intensity time series alone.

\section{Conclusion}

Motivated by the effectiveness of the discrete BDI scheme in the context of electromagnetic scattering, we have extended it to encompass the class of Pearson diffusions as the continuum limit of a discrete population model. This establishes a mathematical basis for a more general class of probability densities for the (texture component of the) scattered amplitude as the asymptotic stationary distributions of these diffusions. Besides the $\Gamma$-distribution for the texture that corresponds to the widespread $K$-distribution for the intensity, only a few instances of the Pearson system yield analytical expressions for the scattering amplitude distribution [4]. Nevertheless, these diffusions enable the extension of the stochastic framework developed in [8] and [9] to include additional intensity distributions which are further characterized by analytical expressions for their autocorrelation functions and power spectra. Experimental time series for the intensity may exhibit temporal correlation patterns more sophisticated than the exponential form (3.2). Nonstationary patterns can be accommodated by considering delays in the transition functions of the rate equation (2.4), which is conceptually equivalent to the introduction of a delay in the SDE (2.9) [13].

We have also shown that the underlying discrete-valued population model is deducible through the intensity time series. This feature can provide some insight into the physical phenomena, yielding a particular intensity distribution pattern. As a parametric generalization of the BDI process (which results in a $K$-distributed noise), the Pearson class could provide a more refined model for scattering data that exhibit a slight deviation from the $K$-distribution (for example, to account for the discrepancy in tails with regards to observed data). Furthermore, the propagator of (2.9) has been derived in [18] for several cases, thus enabling, beyond the already known autocorrelation function, the derivation of all higher-order statistics.

\section{References}

[1] Balleri, A., Nehorai, A. And Wang, J. (2007). Maximum likelihood estimation for compound-Gaussian clutter with inverse gamma texture. IEEE Trans. Aerosp. Electron. Syst. 43, 775-780.

[2] Bartlett, M. S. (1966). An Introduction to Stochastic Processes, with Special Reference to Methods and Applications. Cambridge University Press.

[3] Bewernick, R. L. et al. (2007). On the representation of birth-death processes with polynomial transition rates. J. Statist. Theory Pract. 1, 227-231.

[4] Delignon, Y. And PIECZYNSKI, W. (2002). Modelling non-Rayleigh speckle distribution in SAR images. IEEE Trans. Geosci. Remote Sensing 40, 1430-1435.

[5] Farina, A., Gini, F., Greco, M. and Verrazzani, L. (1997). High resolution sea clutter data: statistical analysis of recorded live data. IEE Proc. Radar Sonar Navig. 144, 121-130.

[6] FAYARD, P. AND FIELD, T. R. (2008). Optimal inference of the scattering cross-section through the phase decoherence. Waves Random Complex Media 18, 571-584.

[7] FIELD, T. R. (2005). Observability of the scattering cross-section through phase decoherence. J. Math. Phys. 46, 063305, 8pp.

[8] Field, T. R. And Tough, R. J. A. (2003). Diffusion processes in electromagnetic scattering generating $K$-distributed noise. Proc. R. Soc. London A 459, 2169-2193.

[9] Field, T. R. AND Tough, R. J. A. (2003). Stochastic dynamics of the scattering amplitude generating K-distributed noise. J. Math. Phys. 44, 5212-5223. 
[10] Forman, J. L. And Sørensen, M. (2008). The Pearson diffusions: a class of statistically tractable diffusion processes. Scand. J. Statist. 35, 438-465.

[11] Jakeman, E. (1980). On the statistics of $K$-distributed noise. J. Phys. A 13, 31-48.

[12] Jakeman, E. And Tough, R. J. A. (1988). Non-Gaussian models for the statistics of scattered waves. Adv. Phys. 37, 471-529.

[13] OHIRA, T. (1997). Oscillatory correlation of delayed random walks. Phys. Rev. E 55, 1255-1258.

[14] ØKsendal, B. (1988). Stochastic Differential Equations: An Introduction with Applications, 5th edn. Springer, Berlin.

[15] Risken, H. (1989). The Fokker-Planck Equation. Springer, Berlin.

[16] WARD, K. (1981). Compound representation of high resolution sea clutter. Electron. Lett. 17, 561-563.

[17] Ward, K. D., Tough, R. J. A. And Watts, S. (2006). Sea clutter: Scattering, the K-distribution and Radar Performance. IET, London.

[18] Wong, E. (1964). The construction of a class of stationary Markov processes. In Proc. Symp. Appl. Math., Vol. 16, American Mathematical Society, Providence, RI, pp. 264-276. 